\section{Using a Bed Sheet to Avoid an Assisted Delivery}

To the Editor: A few years ago, I attended the delivery of a primigravida who had received epidural anesthesia who was nearing delivery after 3 hours of pushing. She was exhausted and no longer pushing effectively. As I contemplated an assisted delivery with a vacuum or forceps, a midwife suggested a technique using a bed sheet. With this method, the patient soon delivered a healthy baby without the need for a vacuum nor forceps. I have successfully employed this simple alternative a number of times since.

The procedure is easy to perform. A knot is tied in both ends of a sheet (Figure 1). One end is held by the patient from a dorsal lithotomy or lateral position, and the other by the maternity care provider from the foot of the bed. With contractions, the provider and patient pull against each other (Figure 2).

This procedure is designed to increase expulsive efforts and improve maternal positioning. As the patient pulls on the sheet, she automatically increases intraabdominal pressure through a Valsalva maneuver. The physical communication through the sheet allows the provider to coach better how long to push, when to breathe and when to relax. Maternal positioning is opti- mized with an automatic flexing of the maternal head and curving around the baby. This technique may be especially useful for patients who cannot be upright because of an epidural or other reason.

Although use of the sheet technique avoids most risks of assisted delivery, it does carry some risks and contraindications. It requires significant physical exertion by the maternity care provider, which may be unsafe or impossible for some. To overcome this, help could be enlisted or the sheet could be tied to the foot of the bed, although the physical coaching aspect would be lessened. Contraindications to performing the sheet technique include: fetal distress and certain maternal conditions such as hemorrhage, any condition contraindicating a Valsalva maneuver, and any condition preventing grasping of the sheet.

I have not been able to find this technique described in the literature. I hope this letter will inspire usage of the technique and encourage well-designed studies to document its effectiveness.

Lee T. Dresang, MD St. Luke's Family Practice Residency Department of Family Medicine University of Wisconsin Medical School Milwaukee

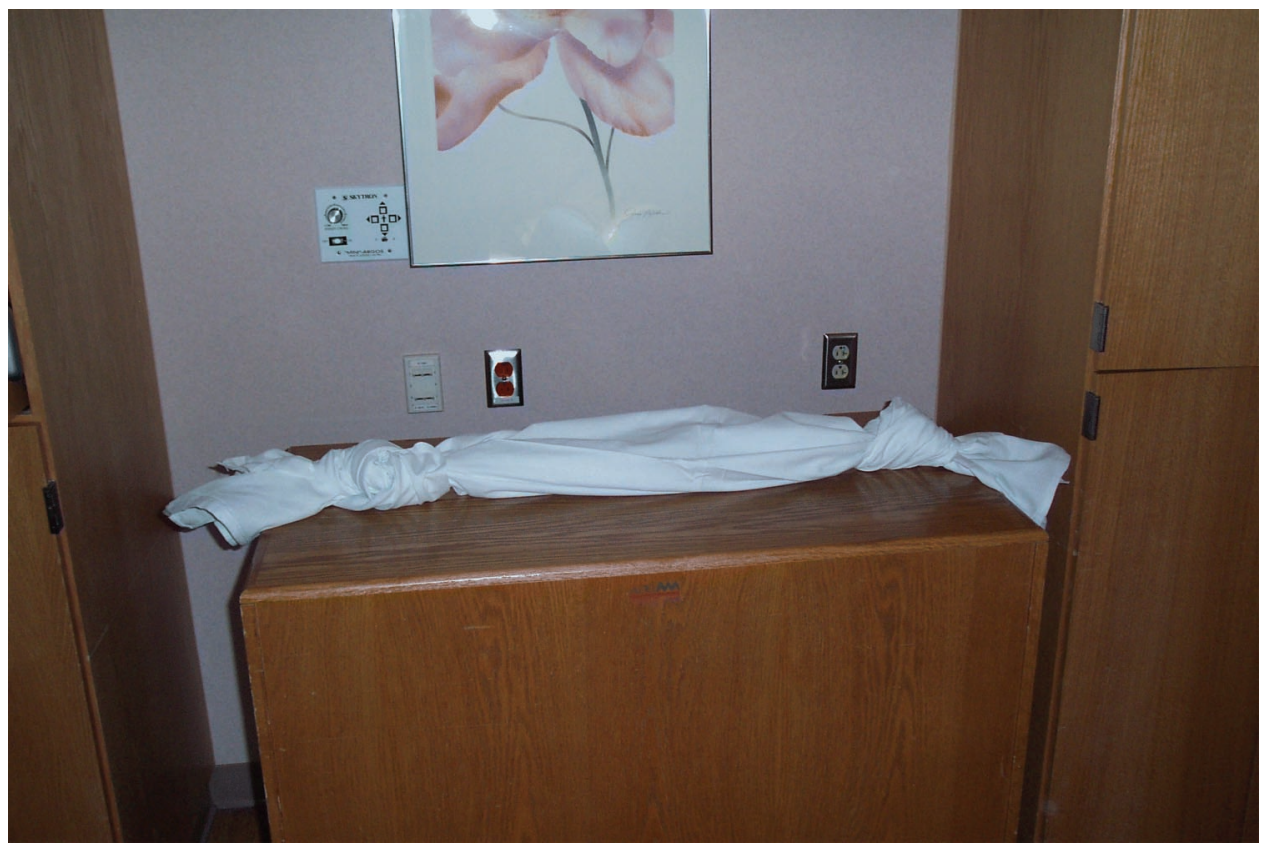

Figure 1. A knot is tied in both ends of a sheet. 


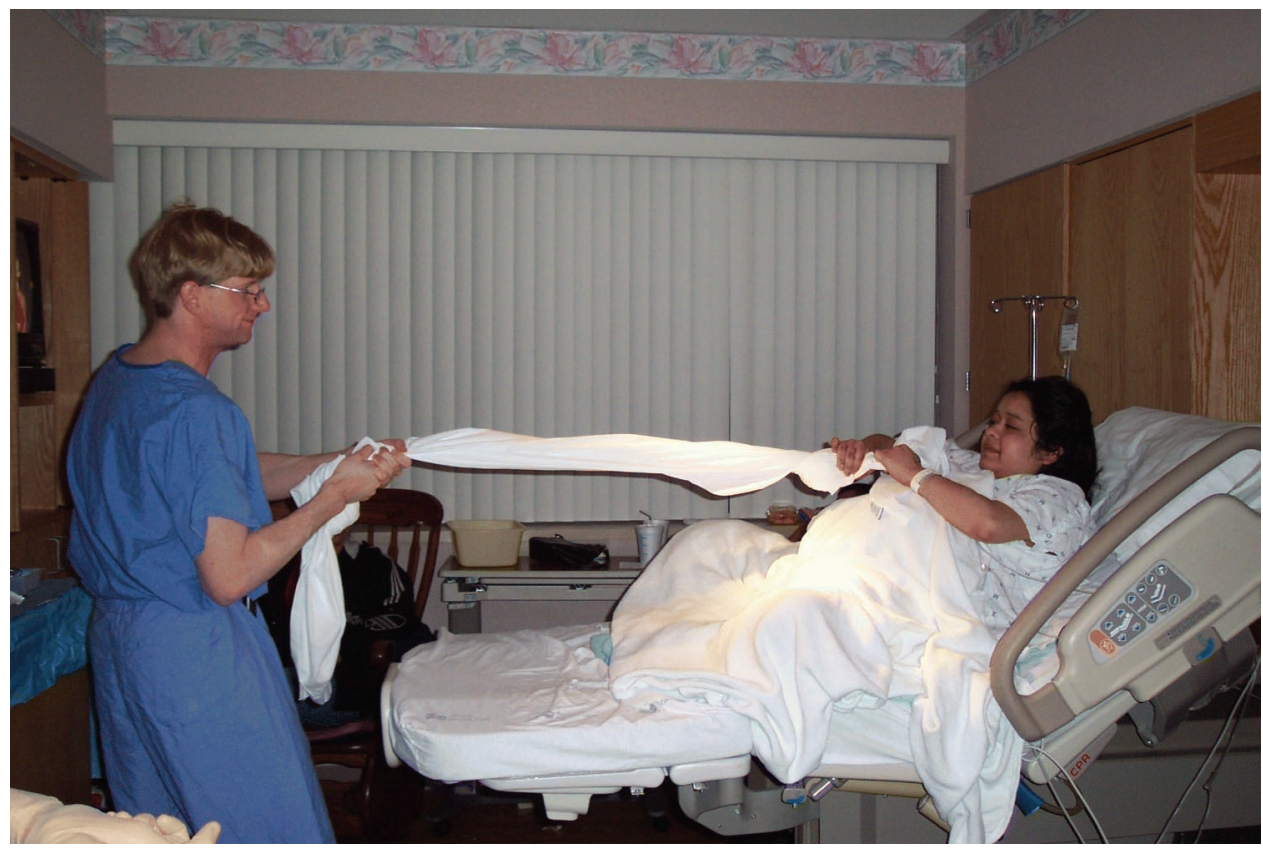

Figure 2. With contractions, the provider and patient pull against each other.

\section{The Variable Location, Content, and Legibility of Expiration Dates on Medicine Containers}

To the Editor: Law mandates expiration-dates on drug products to ensure their "identity, strength, quality and purity." It stipulates that such date "shall appear on the immediate (product) container" without further specifying a particular location. ${ }^{2}$ It is silent about the legibility and content of the date inscription. Besides the medicine's name and strength, users look for this date on a medicine container. To assess the ease of reading and locating these important dates, I evaluated a global sample of all medicines in a traditional, urban family practice office. Descriptors of the 84 study medicines, marketed by 49 US companies, were formulation (oral, 44; topical, 23; injection, 10; inhaler, 7), source (samples, 68; purchased, 16), and nature (brand, 70; generic, 14).

Expiration-date locations were: container bottom 27 $(32 \%)$, side $24(29 \%)$, rear 14 (17\%), front $12(14 \%)$, and top $7(8 \%)$. Date formats were: month-year, 76 ; daymonth-year, 5; month-day-year, 2; and year-month, 1 (Figure 3). One inscription read "02/04," which could mean either February 2004 or April 2002 (Figure 3, \#3). The dates were preceded by the following captions: "Exp.", 53; "Exp. Date", 16; "Ex", 1; none, 14 (Figure 3). On 11 packages, dates were printed in landscape while the adjacent text was in a portrait or inverse orientation (Figure 3, \#2). For 3 inhalers, an opaque dispenser required removal to see the date on the canister.

Legibility was good for $36(80 \%)$ and fair for $9(20 \%)$ of the 45 printed inscriptions. Among the 39 embossed dates, legibility was good for 8 (21\%), fair for 16 (41\%), and poor for 15 (38\%); it was impossible to decipher the full date in one case (Figure 3, \#6 to 8). Causes of poor legibility were superficial embossment, inadequate contrast between letters and background (due to lack of ink), glare from 3-dimensional carving (worse on glossy surfaces or a dark background), dot matrix printing, small font size, and embossment on ointments' narrow (2- to 4-mm wide) crimps. These problems discouraged one clinic physician from accepting or dispensing the samples. Samples of three medicines (14 containers) had passed their expiration dates and required discarding.

The expiration date location on medicine containers varies. This forces the user to look for it by turning the container around. Such search can be annoying to physicians who dispense several medicine samples daily. Although the date-search requires only a few seconds, such time may be critical in an emergency, or a busy schedule, particularly as it nips into the physician's face-to-face interaction with the patient. The date location on medicine containers requires standardization. Ideally, it should be on the container's front, directly above or below the medicine's name; this would eliminate the need to turn the container to check multiple surfaces. To avoid confusion or distraction, this area should contain few other numbers. Dating should follow the national convention: month-day-year in the United States, daymonth-year in the United Kingdom. A month-year format causes waste because uninformed consumers may discard a medicine on the month's first day although it officially expires on the last. ${ }^{3}$ For inhalers, the date should be printed on that canister's bottom (the end that remains visible after dispenser assembly), or the dis- 

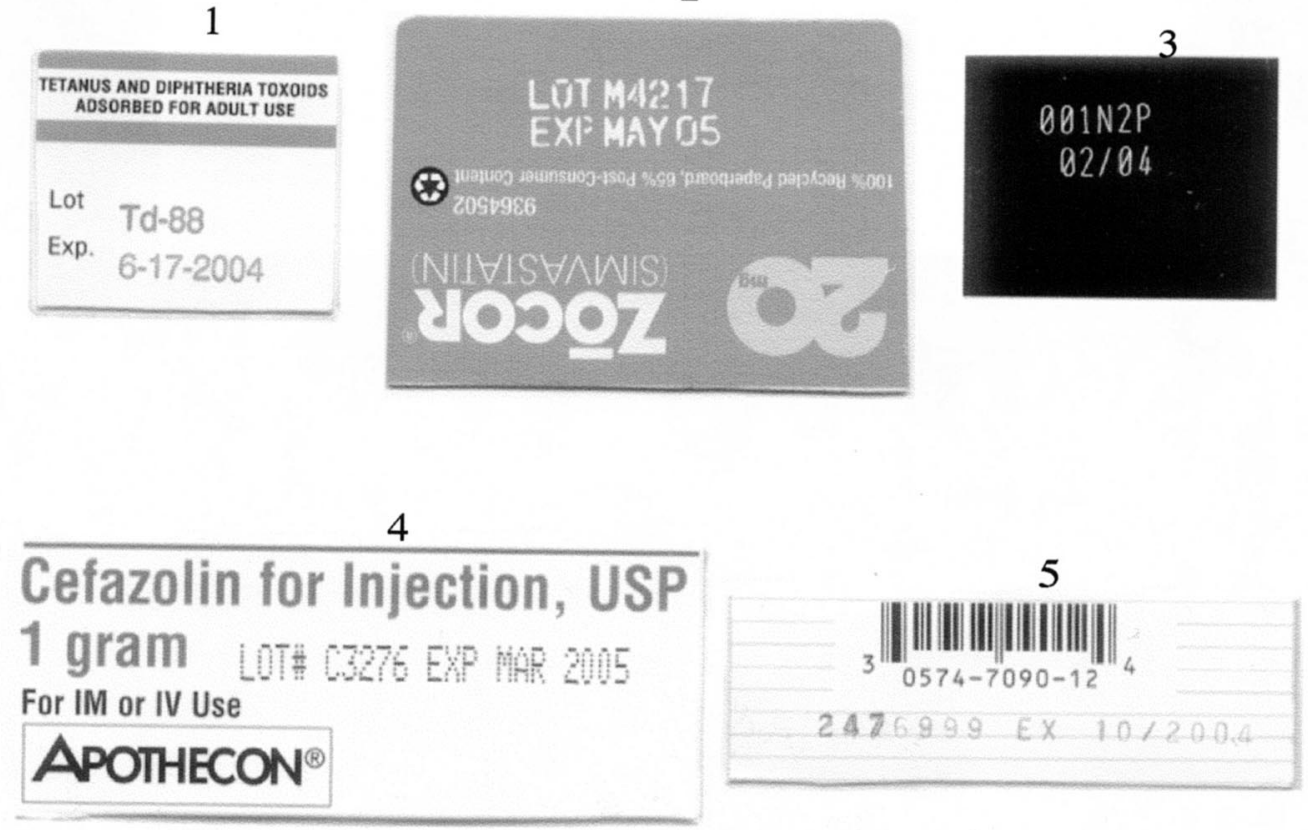

7

6

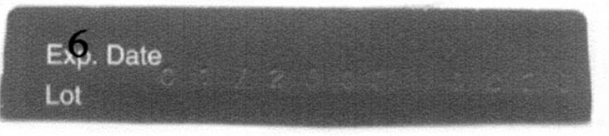

Lot No. and Exp. Date

\section{Font Sizes: 67891011121314151617181920}

Figure 3. Expiration-date inscriptions illustrating variable legibility, format (standard, inverse), background (black, white, color), caption (none, "Exp. Date," "Exp"), printing (dot matrix, laser), and content (month-year, month-date-year, date-month-year). Notice the poor legibility of the embossed inscriptions (\#6 to 8). Numbered fonts are shown at the bottom for letter size comparison.

penser should be transparent. A standardized caption (eg, "Exp. Date," "Expires") should always precede the date.

To improve legibility, dates should be printed in black or red ink against a white background, in a common font (eg, Times New Roman) of modest ( $>10$ points) size, on a non-glare surface, and not on ointment crimps. On smaller containers (eg, eye/ear drops, injections), necessary space for a legible date should be found by moving less consumer-relevant information (eg, lot number, manufacturer name) to another surface. Good legibility is crucial because millions of medicine-users are older persons with presbyopia.

Conventional research wisdom might cast some doubt on the findings of this study as it was based on observations in one single medical practice. However, the study site was typical of others in the community, it did not manufacture any of the medicines, it was not affiliated with any drug manufacturer, and the medicines came from a large number of US companies.

The law requires physicians to exercise the same level of care as pharmacists when dispensing medicines. ${ }^{4}$ Thus, physicians must check the expiration-date on each individual medicine sample container. Any efficiency that makes this financially nonremunerative date-reading task less cumbersome will enhance sample dispensing by physicians, reduce waste, and thus even facilitate the manufacturers' marketing effort. This study highlights real-life problems, and proposes simple remedies. Its recommendations should apply also to nonprescription medicines and other dated items, such as food products. Industry 
should modify its date-inscribing practice so that human perfection is not required to find and read the dates. To paraphrase Alice in Wonderland, what good is an inscription "if I can't read it!"

Sapna Lohiya, MD Royal Medical Group

Costa Mesa, CA

\section{References}

1. Expiration Dating. 21 C.F.R. Sect. 211.137 (1995).

2. Expiration Dating. 21 C.F.R. Sect. 201.17 (1995).

3. US Pharmacopeia/National Formulary. 22nd ed. Rockville (MD): US Pharmacopeial Convention Inc.; 1985. p. 9.

4. Medical Board of California. Legal requirements for distribution of drug samples. Action Report 1996;56:3. 